\section{Ten years of tension: single-molecule DNA mechanics}

\author{
Carlos Bustamante ${ }^{\star} \uparrow$, Zev Bryant ${ }^{\star}$ \& Steven B. Smith $\uparrow$
}

${ }^{*}$ Department of Molecular and Cell Biology, and $\dagger$ Department of Physics and Howard Hughes Medical Institute, University of California, Berkeley, California94720,USA (e-mail: carlos@alice.berkeley.edu; zev@alice.berkeley.edu; steve@alice.berkeley.edu)

The basic features of DNA were elucidated during the halfcentury following the discovery of the double helix. But it is only during the past decade that researchers have been able to manipulate single molecules of DNA to make direct measurements of its mechanical properties. These studies have illuminated the nature of interactions between DNA and proteins, the constraints within which the cellular machinery operates, and the forces created by DNA-dependent motors.

\section{T} he physical properties of the DNA double helix are unlike those of any other natural or synthetic polymer. The molecule's characteristic base stacking and braided architecture lend it unusual stiffness: it takes about 50 times more energy to bend a double-stranded DNA (dsDNA) molecule into a circle than to perform the same operation on singlestranded DNA (ssDNA). Moreover, the phosphates in DNA's backbone make it one of the most highly charged polymers known.

The protein machinery involved in copying, transcribing and packaging DNA has adapted to exploit these unique physical properties (see article by Alberts, pages 431). For example, RNA polymerases (which synthesize RNA from a DNA template) and helicases (which unwind the double helix to provide single-stranded templates for polymerases) have evolved as motors capable of moving along torsionally constrained DNA molecules. DNA-binding proteins can use the polymer's electrostatic potential to cling to DNA while they diffuse along the molecule in search of their target sequences. Topoisomerases break and rejoin the DNA to relieve torsional strain that accumulates ahead of the replication fork.

During the past ten years, direct manipulation of single molecules of DNA has expanded our understanding of the mechanical interactions between DNA and proteins, following a pattern in which basic investigations of DNA elasticity have laid the groundwork for real-time, single-molecule assays of enzyme mechanism.

\section{DNA as a worm-like chain}

Although mechanical properties vary according to local sequence and helical structure, the relevant physics of DNA in many biological contexts is usefully described using a coarse-grained treatment such as the worm-like chain (WLC) model $^{1}$, which characterizes a polymer using a single parameter, the flexural persistence length $(A)$. The WLC model imagines a polymer as a line that bends smoothly under the influence of random thermal fluctuations. The value of $A$ defines the distance over which the direction of this line persists: correlation between the orientations of two polymer segments falls off exponentially (with decay length $A$ ) according to the contour length that separates them. For dsDNA in physiological buffer, $A=\sim 50 \mathrm{~nm}$.

There is a simple relationship between $A$ and the bending rigidity $\kappa$ of the polymer represented as an elastic $\operatorname{rod}^{2}: k_{\mathrm{B}} T A=\kappa$, where $k_{\mathrm{B}}$ is
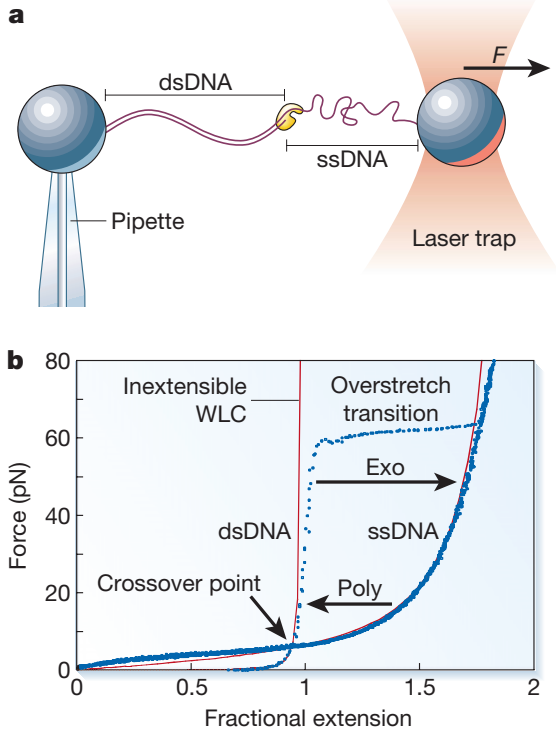

Figure 1 Single-molecule assays of replication ${ }^{12,13} \cdot \mathbf{a}$, A DNA molecule is stretched between beads held in a micropipette and a force-measuring optical trap ${ }^{12}$. The measured extension is the sum of contributions from the single-stranded DNA (ssDNA) and double-stranded DNA (dsDNA) segments. b, Force versus extension for dsDNA and ssDNA molecules, obtained in the instrument in panel a. Arrows show changes in extension observed at constant tension during polymerization (Poly) or force-induced exonuclease activity (Exo).

Boltzmann's constant and $T$ is the temperature. According to this relation, the energy required to bend a segment of DNA of length $L$ through an angle $\theta$ and a radius of curvature $R / L$ is:

$$
E(\theta)=\frac{k_{\mathrm{B}}}{2 R^{2}}=\frac{k_{\mathrm{B}}}{2 L} \frac{T A}{{ }^{2}} \theta^{2}
$$

This model, therefore, predicts that it is energetically more favourable to bend the molecule smoothly, spreading the strain over large distances, than to bend it sharply at discrete locations. This mechanical property is central to interactions with regulatory proteins that bend DNA severely upon binding. The biological relevance of these bends is demonstrated by the enhancement of DNA recombination and gene transcription observed when specific protein-binding sites for activators are replaced by intrinsically bent DNA sequences ${ }^{3}$ or by binding sequences for unrelated DNAbending proteins in the presence of these proteins $s^{4}$.

To bend DNA, proteins must convert part of their binding energy into mechanical work, as illustrated by an experiment in which a binding sequence was pre-bent towards the major groove by placing it in a DNA minicircle. The affinity of a transcription factor (TBP) for this binding site was found to be 300 -fold higher (equivalent to a freeenergy change of $3.4 \mathrm{kcal} \mathrm{mol}^{-1}$ ) when the sequence was pre-bent in the same direction as TBP-induced bending, relative to pre-bending in the opposite direction ${ }^{5}$. This increase can largely be accounted for by the difference in bending energy between the two initial DNA conformations, which by the equation above is predicted to be $3.2 \mathrm{kcal} \mathrm{mol}^{-1}$.

The high linear charge density of the double helix provides one mechanism for converting binding energy into work. DNA's structure is pre-stressed by electrostatic self-repulsion, as a result of the negatively charged phosphate backbone of the double helix. Therefore, asymmetric neutralization of the DNA helix (for example, by a DNAbinding protein that presents a positively charged face) can lead to compression and bending of DNA towards the neutralized face. This 
a
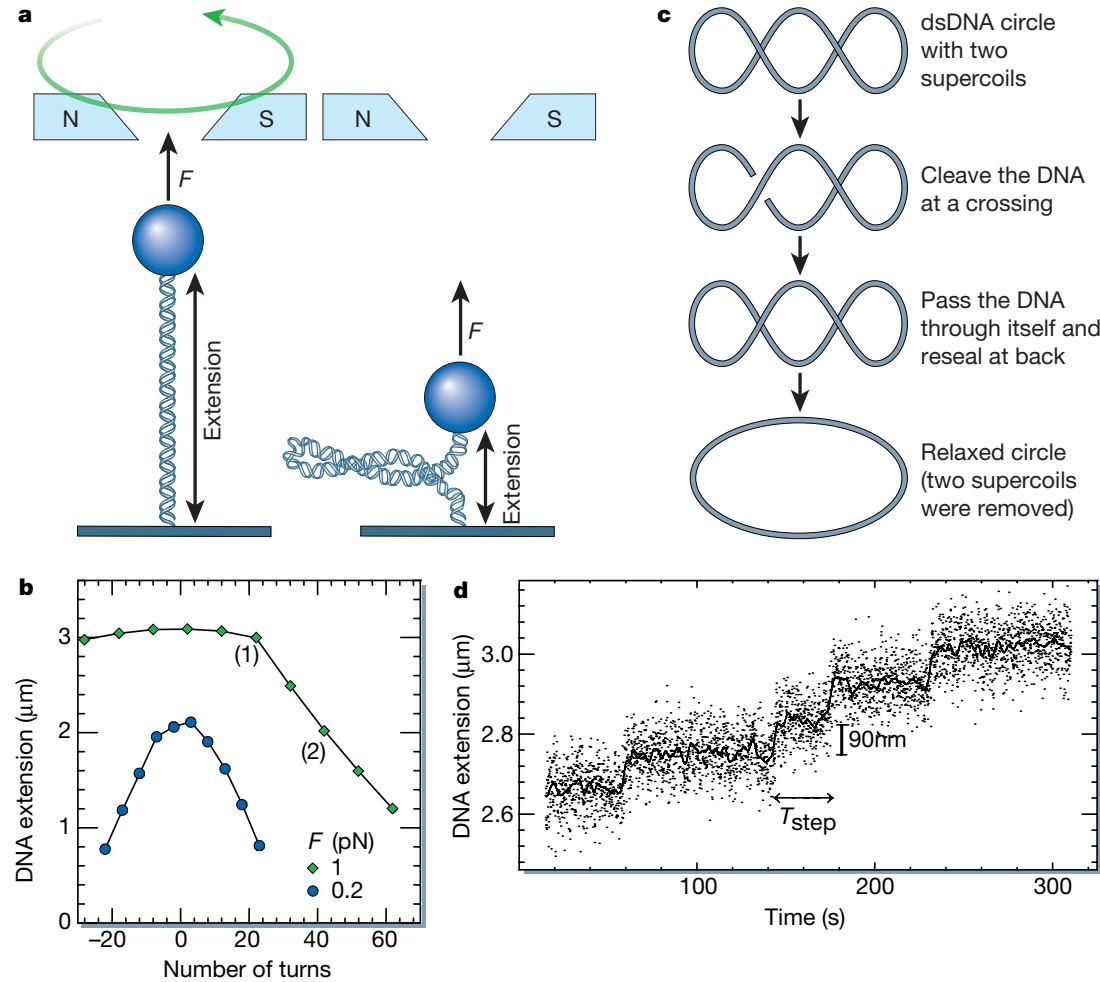

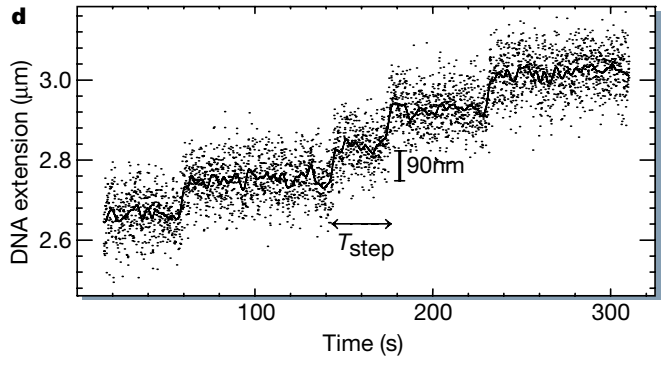

Figure 2 The elastic behaviour of supercoiled DNA molecules ${ }^{49}$ forms the basis of singlemolecule topoisomerase assays ${ }^{15-17}$. a, Molecules are stretched and twisted in magnetic tweezers. Under sufficient torsiona strain, a twisted DNA molecule buckles to form plectonemes, shortening the measured extension. b. Extension as a function of turns introduced into the molecule remains nearly constant until the buckling transition is reached (1), after which the molecule contracts linearly (2). Underwinding at $1 \mathrm{pN}$ does not cause reduced extension because the strained molecule forms alternative underwound structures (for example, melted DNA) in preference to buckling. c, The accepted model of type II topoisomerase action: the enzyme binds to supercoiled DNA, cleaves both strands, and passes the double helix through itself, leading to the removal of two turns. (Redrawn from ref. 50, with permission.) $\mathbf{d}$, When topoisomerase $\|$ is added to a plectonemed molecule in the magnetic tweezers, 90-nm steps in extension are seen, corresponding to the removal of two turns ${ }^{15}$, as predicted by the model. effect has been elegantly demonstrated by incorporating neutral phosphate analogues or tethered cations onto one face of a DNA molecule ${ }^{6}$.

\section{DNA elasticity}

The bending elasticity of DNA has consequences beyond short-range interactions with proteins: the WLC model explicitly connects local bending mechanics with the statistics of global conformations. Thus, a polymer with smaller bending rigidity tends to adopt a more compact random-coil structure.

This preference is reflected in the phenomenon of entropic elasticity, which is responsible for the elastic properties of common polymeric materials such as rubber ${ }^{7}$. A flexible polymer coils randomly in solution, resulting in an average end-to-end distance much shorter than its contour length. Pulling the molecule into a more extended chain is entropically unfavourable, as there are fewer possible conformations at longer extensions, with only a single possible conformation (a perfectly straight line) for maximum extension. The resulting entropic force increases as a random coil is pulled from the ends; tensions on the order of $k_{\mathrm{B}} T / A(\sim 0.1 \mathrm{pN}$ for dsDNA or $5 \mathrm{pN}$ for ssDNA) are required to extend the molecule significantly.

Direct measurements of force and extension on single molecules of DNA provide the most rigorous test to date of theories of entropic elasticity. When magnets and fluid flow ${ }^{8}$ and later optical traps $s^{9,10}$ were used to stretch DNA molecules attached to micron-scale beads (Fig. 1), the entropic force-extension behaviour of dsDNA was found to agree closely with the WLC model ${ }^{11}$. Tensions of $\sim 6 \mathrm{pN}$, within the range of forces exerted by characterized molecular motors, stretch dsDNA to $~ 95 \%$ of its contour length.

The intrinsic flexibility of ssDNA causes it to maintain very compact conformations, so that its extension per base pair is shorter than that of dsDNA for forces smaller than $\sim 6 \mathrm{pN}$. At higher forces, however, the situation is reversed. A single strand is not constrained to follow a helical path, so it becomes nearly twice as long as dsDNA as it is pulled straight (Fig. 1).

\section{From elasticity to enzymology}

A quantitative appreciation of the different elastic properties of
ssDNA and dsDNA has allowed researchers to observe replication of single DNA molecules ${ }^{12,13}$. In these studies, a molecule of ssDNA was stretched between two surfaces, and a DNA polymerase was allowed to replicate the stretched molecule at a given constant tension. As ssDNA was converted into dsDNA by the polymerase, replication could be followed in real time by monitoring the extension (below $6 \mathrm{pN}$ ) or contraction (above $6 \mathrm{pN}$ ) of the molecule (Fig. 1).

These studies showed that the rate-limiting step of DNA replication, which involves closing a structural 'fingers' domain of the enzyme, is sensitive to DNA tension and is capable of generating forces as high as $35 \mathrm{pN}$. Small forces can accelerate the enzyme's activity, probably by helping it to stretch the proximal collapsed template strand into the correct geometry for polymerization. A surprising result was the induction of a strong exonuclease activity (removal of nucleotides) at tensions above $40 \mathrm{pN}$ (ref. 12). This effect provides a novel assay to investigate the proofreading mechanism of DNA polymerases.

Studies of the force-extension behaviour of single supercoiled DNA molecules further illustrate the progression from elasticity measurements to enzymology (Fig. 2). DNA tethers were stretched between a surface and a magnetic bead that could be rotated using magnets ${ }^{14}$. Because the molecule was attached at each end through multiple linkages on both strands, rotation of the bead led to the build up of torsional strain in the molecule. Under tension, such a molecule behaves roughly like a twisted rubber tube: as turns are added, the extension remains nearly constant until a critical amount of torque accumulates and the tube buckles, trading twist for writhe to form plectonemes (units of supercoiled DNA that project out of the molecular axis), thus reducing its apparent extension with each subsequent turn. As tension is increased, so does the energetic penalty for buckling; therefore, more turns must be added to reach the buckling transition.

The activity of topoisomerase II, an enzyme that relaxes supercoils in eukaryotic cells, has been analysed on single plectonemed DNA teth$\mathrm{ers}^{15}$. Under conditions of limiting ATP, discrete steps in extension were observed that were attributable to single enzymatic turnovers. The size of these steps corresponded to the removal of two turns, confirming the 


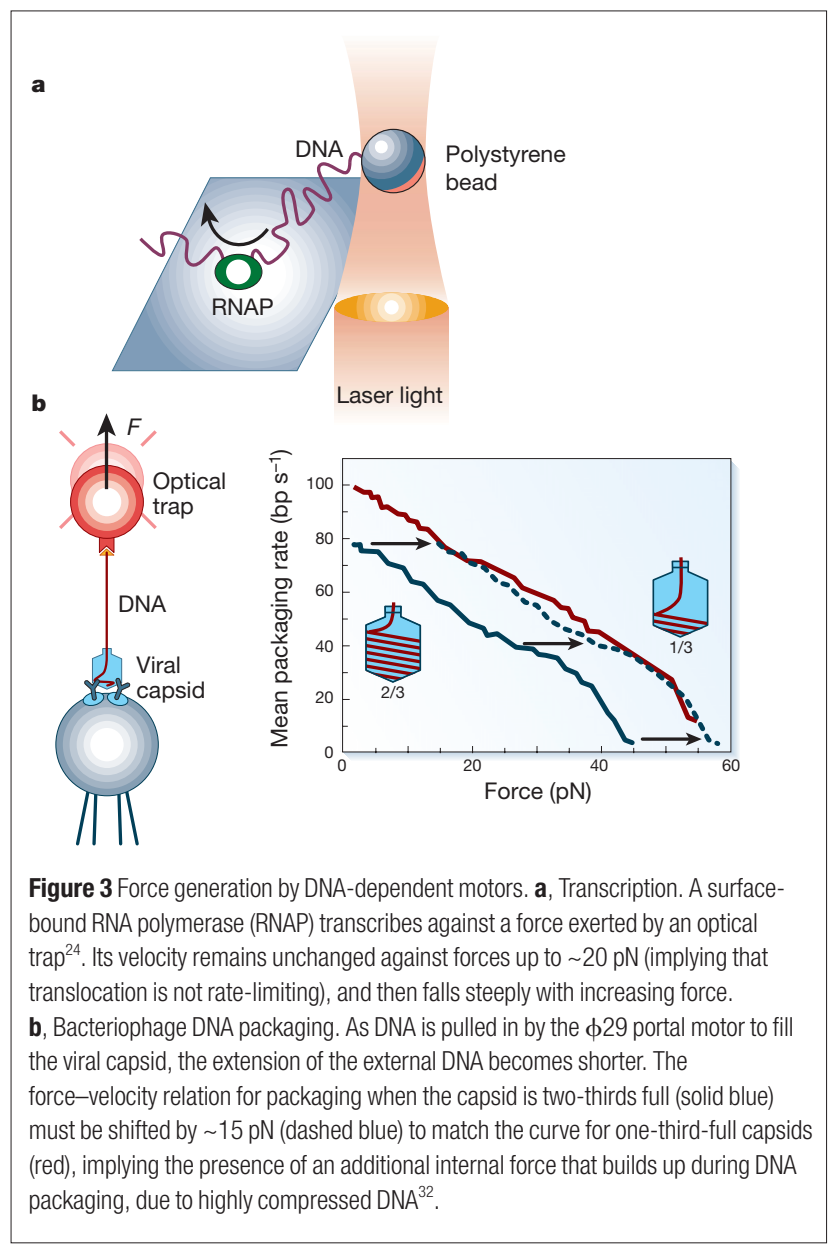

accepted model of topoisomerase II action in which the double helix is passed through itself, changing the linking number by two (Fig. 2). Later experiments applied the same methodology to bacterial topoisomerases I and IV (refs 16 and 17, respectively).

Single-molecule assays of topoisomerase IV (ref. 17) helped reveal that the enzyme has a chiral substrate specificity: it relaxes overwound DNA substantially more efficiently than underwound DNA. This allows the enzyme to relax the positive supercoils formed during replication while avoiding counterproductive relaxation of the negative supercoils present in non-replicating DNA.

These studies also showed that topoisomerase IV relaxes DNA (of either handedness) an order of magnitude faster than had been estimated from bulk studies, helping to resolve a dilemma in which the enzyme's apparent low turnover rate in vitro had seemed to be at odds with its demonstrated ability to counteract rapid supercoil formation at the replication fork in vivo. This result reflects a general caveat for bulk enzyme kinetics: the presence of inactive enzymes in solution can lead to gross underestimation of the turnover rate per enzyme. Single-molecule assays, including those based on DNA manipulation, can sidestep this issue by selecting only the active fraction for analysis.

\section{DNA unzipped}

DNA helicases must generate force to unzip the parental strands during replication (see article by Alberts, page 431). Mechanical unzipping forces for dsDNA were first measured by attaching one strand to a surface (through a dsDNA linker) and pulling on the other strand using a glass needle ${ }^{18}$ or optical tweezers ${ }^{19}$ as a force transducer. DNA from a bacterial virus, called lambda phage, was unzipped (and re-zipped) at forces between 10 and $15 \mathrm{pN}$, depend- ing on the local sequence. The pattern of force fluctuations during unzipping of a particular sequence was remarkably reproducible, and could be rationalized from a simple model incorporating the known difference in stability between adenine-thymine and guanine-cytosine base pairs.

Future studies might use this experimental geometry to investigate helicase function directly. Such an experiment could provide insight into the rates, processivity, force generation and sequence dependence of helicases, complementing the results of previous single-molecule helicase assays which observed translocation without measuring or applying forces ${ }^{20,21}$.

In a new application of mechanical unzipping ${ }^{22}$, the extra force needed to separate the DNA strands past DNA-binding proteins has been used to map the positions of target sequences, and (by noting the fraction of occupied sites as a function of protein concentration) to measure dissociation constants.

\section{Forces in DNA transcription and packaging}

The ability to apply forces on DNA has altered the way we think about DNA-dependent enzymes, by revealing these enzymes to be powerful motors (Fig. 3). Optical tweezers have been used to follow transcription by Escherichia coli RNA polymerase against external loads ${ }^{23-25}$. This enzyme can generate forces exceeding those of cytoskeletal motors that drive transport processes within the cell ${ }^{26}$. Its velocity remains unchanged against forces of up to $\sim 20 \mathrm{pN}$ (refs 24,25), showing that the translocation step (which must by definition be force sensitive) is not rate limiting.

An externalload can, however, affect the tendency of an enzyme $e^{25}$ to pause or arrest during transcription. The application of force in an 'aiding' direction ${ }^{27}$ reduces pausing and arrest probabilities, presumably by preventing the polymerase from sliding backwards along the template during entry into these inactive states ${ }^{28}$. The same 'backsliding' phenomenon may be responsible for the steep drop in transcriptional velocity seen as opposing force is increased above $\sim 20 \mathrm{pN}$ (ref. 24).

In eukaryotes, forces generated by RNA polymerase or by chromatin-remodelling enzymes might help to displace nucleosomes that would otherwise impede transcription. In support of this idea, several groups have pulled on single chromatin fibres and found that nucleosomes can be removed from DNA by applying a tension of $\sim 20 \mathrm{pN}$ (refs $29-31)$. At lower forces $(\sim 6 \mathrm{pN})$, reversible modifications of the chromatin structure (such as partial DNA release ${ }^{31}$ or disruption of internucleosomal interactions ${ }^{29}$ ) are observed. These tension-inducible structural rearrangements might be exploited by RNA polymerase or other cellular factors to modulate access of the transcriptional apparatus to chromosomal DNA.

The machine that packs DNA into the viral capsid of the bacteriophage $\phi 29$ (a virus that infects bacteria) generates higher forces than have been seen so far for any other translocating (displacing) molecular motor $^{32}$ (Fig. 3). A comparison of the force-velocity relation of the motor when the capsid is mostly empty with that when it is nearly full of DNA revealed the presence of a large internal force (up to $\sim 50 \mathrm{pN}$ ) pushing back on the motor. This pressure, which must be overcome in order to package the viral genome, presumably arises from the combined effects of configurational entropy loss, elastic bending energy, electrostatic self-repulsion, and changes in hydration of the DNA upon packaging. The potential energy thus stored by the motor in the form of a pre-compressed 'spring' should provide some of the driving force for DNA ejection into the bacterial cytoplasm when the virus infects.

\section{Extreme forms of DNA}

The helical structure of DNA is highly adaptable and can assume various forms ${ }^{33}$. Although the helix of dsDNA is typically righthanded and extended in aqueous solution (B form), it can become shorter and wider (A form) in dehydrating solution. Molecules with specific base sequences (alternating purines and pyrimidines) easily assume the left-handed $\mathrm{Z}$ form, which is longer than B form and has reverse twist. Recently, single-molecule manipulation experiments 

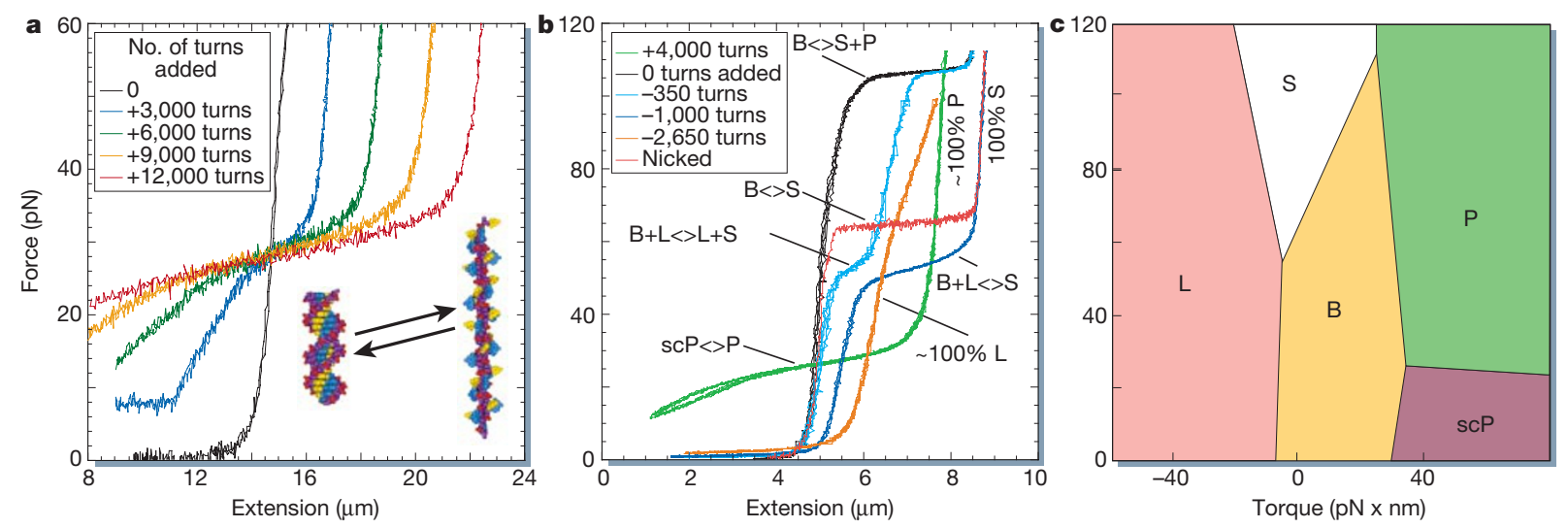

Figure 4 Mechanically induced structural transitions in twist-constrained DNA. a, Force-extension curves for a 44.4-kilobase DNA molecule overwound by successively larger numbers of turns (Z.B., M. D. Stone, S.B.S., N. R. Cozzarelli and C.B., unpublished data). As was seen in ref. 39, the high-force curves can by interpreted as the sum of contributions from a fraction of the molecule that remains in $\mathrm{B}$ form and a progressively larger fraction that is converted to 'P form', whose structure (proposed from molecular mechanics modelling ${ }^{39}$ ) is shown in the inset (courtesy of R. Lavery). The curves cross at an 'isosbestic point' marking the force at which B-DNA and P-DNA have equal extensions. $\mathbf{b}$, Multiple plateaux occur in force-extension curves for torsionally constrained DNA ${ }^{35}$. (Data shown are for a 14.8-kilobase molecule twisted and stretched in $100 \mathrm{mM} \mathrm{Na}^{+}$; Z.B., M. D. Stone, S.B.S., N. R. Cozzarelli and C.B., unpublished data.) C, These multiple plateaux can be explained by a 'phase diagram' for DNA under torque and tension (adapted from ref. 41). Coloured regions represent conditions under which pure phases occur; lines indicate conditions for phase coexistence within a molecule. S, overstretched; P, Pauling, sc, supercoiled (shortened by forming plectonemes). $\mathrm{L}$ is used here in place of ' $\mathrm{Z}$ '35,41 to denote a phase with an average left-handed twist. Other studies have concluded that this form contains exposed bases, consistent with melted DNA ${ }^{49}$; a mixture of non-canonical forms may in fact be present. A nicked DNA molecule (red curve in $\mathbf{b}$ ) remains at zero torque and therefore crosses the B-S coexistence line at $65 \mathrm{pN}$. have revealed the existence of additional helical forms of DNA stabilized by external forces and torques (Fig. 4).

When tension in a nicked DNA molecule is increased to $65 \mathrm{pN}$, it displays a reversible, cooperative transition to an extended form that is $\sim 70$ per cent longer than normal B-DNA ${ }^{9,34}$ and with substantially reduced twist ${ }^{35}$ (Figs 1b, 4b). But, what is the form of this overstretched dsDNA? Do the strands associate in some specific base-paired structure, dubbed ' $S$ form ${ }^{9,34}$, or does overstretched DNA simply comprise two independent strands of $s s \mathrm{DNA}^{36}$ ? Evidence exists for both models, so the question remains open; a further challenge in single-molecule mechanics is the development of methods to probe the high-resolution structure of manipulated molecules ${ }^{37}$.

Twisting of stretched DNA can lead to other structural transitions ${ }^{35,38,39}$. For example, after a critical amount of overwinding has been introduced into a molecule (Fig. 4a), it gets progressively longer with additional twisting, implying cooperative conversion to an overextended form with greatly increased helicity ( $\sim 2.6$ base pairs per turn, compared with 10.5 base pairs per turn for B-DNA). The evidence $^{39}$ suggests an inside-out double helix reminiscent of the structure proposed by Linus Pauling in 1953 (ref. 40) and therefore dubbed Pauling DNA (P-DNA).

Complex force-extension curves with multiple force plateaux are seen when single DNA molecules are twisted in either direction and pulled to high forces (ref. 35 and Fig. 4b). A simple model to account for these features assumes that DNA has five interconvertible structural forms ${ }^{41}$. This model predicts a force-torque 'phase diagram'(Fig. 4c), thus framing mechanically induced structural transitions in terms of coexistence lines, critical stresses, and triplepoints. Such a model might be tested by direct measurements of torque on stretched and twisted DNA — so far, this quantity has been inferred only indirectly from force-extension experiments. It remains to be determined whether molecular motors can generate sufficient concomitant torque and tension to generate extreme forms' of DNA in a biological context.

\section{From mechanics to nanotechnology}

Single-molecule manipulation of DNA has illuminated the mechanical basis of interactions between DNA and the molecular machinery involved in transcription, replication and recombination. Over the next decade, these studies are likely to expand to include detailed analyses of the mechanical interactions of many factors involved in these fundamental cellular processes. Because of the potentially large class of motors that track the DNA helix (as demonstrated elegantly for RNA polymerase ${ }^{42}$ ), necessary technical improvements will include direct measurement of torque in experiments that decouple twisting from bending.

Outside of traditional DNA biology, the ease of synthesis and well-characterized elasticity of DNA make it an ideal material for stiff molecular 'handles' to manipulate other molecules. So far, such handles have been used to mechanically unfold molecules of $\mathrm{RNA}^{43}$, but covalent attachment of DNA segments to protein molecules has also been demonstrated ${ }^{44}$, opening the door for the next generation of forced protein (un)folding studies ${ }^{45}$ and perhaps mechanical assays of domain motion in enzymes.

Engineers have recently exploited the properties of DNA to construct self-assembled nanomachines, such as artificial DNA-based devices driven by strand displacement ${ }^{46,47}$ or chemically induced structural rearrangements (ref. 48; and see article by Seeman, page 427). DNA micromanipulation techniques will help assess the utility of this new class of molecular machines for which force and torque generation have yet to be measured. The past decade has provided a new perspective of the mechanical nature of the double helix. The next decade promises deeper insight into its interactions with the cellular machinery and its potential for constructing sophisticated nanomachines.

\section{doi:10.1038/nature01405}

1. Kratky, O. \& Porod, G. Röntgenuntersushung gelöster Fagenmoleküle. Rec. Trav. Chim. Pays-Bas 68 1106-1123 (1949).

2. Schellman, J. A. Flexibility of DNA. Biopolymers 13, 217-226 (1974).

3. Goodman, S. D. \& Nash, H. D. Functional replacement of a protein-induced bend in a DNA recombination site. Nature 341, 251-254 (1989).

4. Perez-Martin, J. \& Espinosa, M. Protein-induced bending as a transcriptional switch. Science 260, 805-807 (1993).

5. Parvin, J. D., McCormick, R. J., Sharp, P. A. \& Fisher, D. E. Pre-bending of a promoter sequence enhances affinity for the TATA-binding factor. Nature 373, 724-727 (1995).

6. Strauss, J. K. \& Maher, L. J. III DNA bending by asymmetric phosphate neutralization. Science 266, 1829-1834 (1994) 
7. Beuche, F. Physical Properties of Polymers (Interscience, New York, 1962).

8. Smith, S. B., Finzi, L. \& Bustamante, C. Direct mechanical measurements of the elasticity of single DNA molecules by using magnetic beads. Science 258, 1122-1126 (1992).

9. Smith, S. B., Cui, Y. \& Bustamante, C. Overstretching B-DNA: the elastic response of individual double-stranded and single-stranded DNA molecules. Science 271, 795-799 (1996).

10. Wang, M. D., Yin, H., Landick, R., Gelles, J. \& Block, S. M. Stretching DNA with optical tweezers. Biophys. J. 72, 1335-1346 (1997).

11. Bustamante, C., Marko, J. F., Siggia, E. D. \& Smith, S. Entropic elasticity of lambda-phage DNA. Science 265, 1599-1600 (1994).

12. Wuite, G. J., Smith, S. B., Young, M., Keller, D. \& Bustamante, C. Single-molecule studies of the effect of template tension on T7 DNA polymerase activity. Nature 404, 103-106 (2000).

13. Maier, B., Bensimon, D. \& Croquette, V. Replication by a single DNA polymerase of a stretched single-stranded DNA. Proc. Natl Acad. Sci. USA 97, 12002-12007 (2000).

14. Strick, T. R., Allemand, J. F., Bensimon, D., Bensimon, A. \& Croquette, V. The elasticity of a single supercoiled DNA molecule. Science 271, 1835-1837 (1996)

15. Strick, T. R., Croquette, V. \& Bensimon, D. Single-molecule analysis of DNA uncoiling by a type II topoisomerase. Nature 404, 901-904 (2000)

16. Dekker, N. H. et al. The mechanism of type IA topoisomerases. Proc. Natl Acad. Sci. USA 99, 12126-12131 (2002)

17. Crisona, N. J., Strick, T. R., Bensimon, D., Croquette, V. \& Cozzarelli, N. R. Preferential relaxation of positively supercoiled DNA by E. coli topoisomerase IV in single-molecule and ensemble measurements. Genes Dev. 14, 2881-2892 (2000).

18. Essevaz-Roulet, B., Bockelmann, U. \& Heslot, F. Mechanical separation of the complementary strand of DNA. Proc. Natl Acad. Sci. USA 94, 11935-11940 (1997).

19. Bockelmann, U., Thomen, P., Essevaz-Roulet, B., Viasnoff, V. \& Heslot, F. Unzipping DNA with optical tweezers: high sequence sensitivity and force flips. Biophys. J. 82, 1537-1553 (2002)

20. Bianco, P. R. et al. Processive translocation and DNA unwinding by individual RecBCD enzyme molecules. Nature 409, 374-378 (2001).

21.Dohoney, K. M. \& Gelles, J. $\chi$-Sequence recognition and DNA translocation by single RecBCD helicase/nuclease molecules. Nature 409, 370-374 (2001).

22. Koch, S. J., Shundrovsky, A., Jantzen, B. C. \& Wang, M. D. Probing protein-DNA interactions by unzipping a single DNA double helix. Biophys. J. 83, 1098-1105 (2002).

23. Yin, H. et al. Transcription against an applied force. Science 270, 1653-1657 (1995).

24. Wang, M. D. et al. Force and velocity measured for single molecules of RNA polymerase. Science $\mathbf{2 8 2}$ 902-907 (1998).

25. Davenport, R. J., Wuite, G. J., Landick, R. \& Bustamante, C. Single-molecule study of transcriptional pausing and arrest by E. coli RNA polymerase. Science 287, 2497-2500 (2000).

26. Vale, R. D. \& Milligan, R. A. The way things move: looking under the hood of molecular motor proteins. Science 288, 88-95 (2000)

27. Forde, N. R., Izhaky, D., Woodcock, G. R., Wuite, G. J. \& Bustamante, C. Using mechanical force to probe the mechanism of pausing and arrest during continuous elongation by Escherichia coli RNA polymerase. Proc. Natl Acad. Sci. USA 99, 11682-11687 (2002).

28. Landick, R. RNA polymerase slides home: pause and termination site recognition. Cell 88, 741-744 $(1997)$

29. Cui, Y. \& Bustamante, C. Pulling a single chromatin fiber reveals the forces that maintain its higherorder structure. Proc. Natl Acad. Sci. USA 97, 127-132 (2000).

30. Bennink, M. L. et al. Unfolding individual nucleosomes by stretching single chromatin fibers with optical tweezers. Nature Struct. Biol. 8, 606-610 (2001).

31. Brower-Toland, B. D. et al. Mechanical disruption of individual nucleosomes reveals a reversible multistage release of DNA. Proc. Natl Acad. Sci. USA 99, 1960-1965 (2002).

32. Smith, D. E. et al. The bacteriophage $\phi 29$ portal motor can package DNA against a large internal force Nature $413,748-752(2001)$.

33. Calladine, C. R. \& Drew, H. Understanding DNA (Academic, London, 1997).

34. Cluzel, P. et al. DNA: an extensible molecule. Science 271, 792-794 (1996).

35. Leger, J. F. et al. Structural transitions of a twisted and stretched DNA molecule. Phys. Rev. Lett. 83 1066-1069 (1999).

36. Williams, M. C., Rouzina, I. \& Bloomfield, V. A. Thermodynamics of DNA interactions from single molecule stretching experiments. Acc. Chem. Res. 35, 159-166 (2002).

37. Wilkins, M. H. F., Gosling, R. G. \& Seeds, W. E. Nucleic acid: an extensible molecule? Nature 167, 759-760 (1951).

38. Strick, T. R., Allemand, J. F., Bensimon, D. \& Croquette, V. Behavior of supercoiled DNA. Biophys. J. 74, 2016-2028 (1998)

39. Allemand, J. F., Bensimon, D., Lavery, R. \& Croquette, V. Stretched and overwound DNA forms Pauling-like structure with exposed bases. Proc. Natl Acad. Sci. USA 95, 14152-14157 (1998).

40. Pauling, L. \& Corey, R. B. A proposed structure for the nucleic acids. Proc. Natl Acad. Sci. USA 39, 84-97 (1953).

41. Sarkar, A., Leger, J. F., Chatenay, D. \& Marko, J. F. Structural transitions in DNA driven by external force and torque. Phys. Rev. E 63, 051903-1-051903-10 (2001)

42. Harada, Y. et al. Direct observation of DNA rotation during transcription by Escherichia coli RNA polymerase. Nature 409,113-115 (2001).

43. Liphardt, J., Onoa, B., Smith, S. B., Tinoco, I. J. \& Bustamante, C. Reversible unfolding of single RNA molecules by mechanical force. Science 292, 733-737 (2001).

44. Hegner, M. DNA Handles for single molecule experiments. Single Mol. 1, 139-144 (2000).

45. Carrion-Vazquez, M. et al. Mechanical design of proteins studied by single-molecule force spectroscopy and protein engineering. Prog. Biophys. Mol. Biol. 74, 63-91 (2000).

46. Yurke, B., Turberfield, A. J., Mills, A. P., Simmel, F. C. \& Neumann, J. L. A DNA-fuelled molecular machine made of DNA. Nature 406, 605-608 (2000).

47. Yan, H., Zhang, X., Shen, Z. \& Seeman, N. C. A robust DNA mechanical device controlled by hybridization topology. Nature 415, 62-65 (2002).

48. Mao, C., Sun, W., Shen, Z. \& Seeman, N. C. A nanomechanical device based on the B-Z transition of DNA. Nature 397, 144-146 (1999).

49. Strick, T., Allemand, J., Croquette, V. \& Bensimon, D. Twisting and stretching single DNA molecules. Prog. Biophys. Mol. Biol. 74, 115-140 (2000).

50. Alberts, B. et al. Molecular Biology of the Cell (Garland, New York, 2002).
DNA in

a material world

Nadrian C. Seeman

Department of Chemistry, New York University, New York 10003, USA

(e-mail:ned.seeman@nyu.edu)

The specific bonding of DNA base pairs provides the chemical foundation for genetics. This powerful molecular recognition system can be used in nanotechnology to direct the assembly of highly structured materials with specific nanoscale features, as well as in DNA computation to process complex information. The exploitation of DNA for material purposes presents a new chapter in the history of the molecule.

“The nucleic-acid 'system' that operates in terrestrial life is optimized (through evolution) chemistry incarnate. Why not use it ... to allow human beings to sculpt something new, perhaps beautiful, perhaps useful, certainly unnatural." Roald Hoffmann, writing in American Scientist, 1994 (ref. 1).

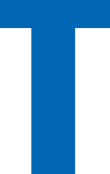

he DNA molecule has appealing features for use in nanotechnology: its minuscule size, with a diameter of about 2 nanometres, its short structural repeat (helical pitch) of about 3.4-3.6 nm, and its 'stiffness', with a persistence length (a measure of stiffness) of around $50 \mathrm{~nm}$. There are two basic types of nanotechnological construction: 'top-down' systems are where microscopic manipulations of small numbers of atoms or molecules fashion elegant patterns (for example, see ref. 2), while in 'bottom-up' constructions, many molecules self-assemble in parallel steps, as a function of their molecular recognition properties. As a chemically based assembly system, DNA will be a key player in bottom-up nanotechnology.

The origins of this approach date to the early 1970s, when in vitro genetic manipulation was first performed by tacking together molecules with 'sticky ends'. A sticky end is a short single-stranded overhang protruding from the end of a double-stranded helical DNA molecule. Like flaps of Velcro, two molecules with complementary sticky ends - that is, their sticky ends have complementary arrangements of the nucleotide bases adenine, cytosine, guanine and thymine - will cohere to form a molecular complex.

Sticky-ended cohesion is arguably the best example of programmable molecular recognition: there is significant diversity to possible sticky ends ( $4^{N}$ for $N$-base sticky ends), and the product formed at the site of this cohesion is the classic DNA double helix. Likewise, the convenience of solid support-based DNA synthesis ${ }^{3}$ makes it is easy to program diverse sequences of sticky ends. Thus, sticky ends offer both predictable control of intermolecular associations and predictable geometry at the point of cohesion. Perhaps one could get similar affinity properties from antibodies and antigens, but, in contrast to DNA sticky ends, the relative three-dimensional orientation of the antibody and the antigen would need to be determined for every new pair. The nucleic acids seem to be unique in this regard, providing a tractable, diverse and programmable system with remarkable control over intermolecular interactions, coupled with known structures for their complexes.

\section{Branched DNA}

There is, however, a catch; the axes of DNA double helices are unbranched lines. Joining DNA molecules by sticky ends can yield longer lines, perhaps with specific components in a particular linear 